\title{
La comercialización sostenible en el refugio de fauna Río Máximo de \\ Camagüey
}

Resumen

María Elena Zequeira Alvarez Santa T. Carrillo Ramos *

El trabajo expone las principales experiencias referidas a la comercialización sustentable en el refugio de fauna Río Máximo, de Camagüey, Cuba. En éste se refleja la armonía existente entre la actividad económica sectorial y la conservación del humedal natural resultado de la utilización de una estrategia ambiental que rige en la cuenca hidrográfica del mismo nombre, la cual integra a todos los tenentes y actores sociales en la zona de amortiguamiento a partir del Plan de Manejo y Operativo del Área Protegida. El artículo forma parte de un estudio de caso del Proyecto GeF/PNUD Sabana Camagüey, dirigido por su Programa de Economía Ambiental. El ecosistema en cuestión es un sitio Ramsar y un área protegida de significación nacional que se distingue por sus valores en la fauna, principalmente por ser el mayor sitio de nidificación del flamenco rosado (Phonicopterus ruber ruber) en la región del Caribe.

Palabras |Cuba, crecimiento económico sostenible, estrategia ambiental,

CLAVE flamenco rosado, refugio de fauna.

*Universidad de Camagüey

***Universidad Central de Las Villas 


\section{Introducción}

El refugio de fauna Río Máximo se localiza en la costa norte del municipio Minas de la Provincia, Camagüey, con coordenadas geográfica 857 I 90 E 225 070 N; 878527 E 225070 N; 878527 E 203445 N y 857 I 90 E 203445 N (Ficha informativa de los Humedales de Ramsar. Categorías aprobadas por la Recomendación 4.7 de la Conferencia de Partes Contratantes).

Cuenta con una altura máxima de cinco metros y una mínima de cuatro. Se caracteriza por el predominio de ecosistemas costeros y marinos-permanente, temporal u ocasionalmente inundados- que incluyen una variedad de hábitats tales como: diversos tipos de bosques siempre verdes semideciduos, manglares, ríos, esteros, canales, ensenadas, lagunas costeras y mares poco profundos que albergan una rica diversidad biológica. Comprende una superficie de 22580 ha (I 4560 ha marinas y 8020 ha terrestres).

Posee gran diversidad de fauna y se distingue por ser el área con mayor sitio de nidificación del flamenco rosado (Phonicopterus ruber ruber) en la región del Caribe. Existen especies endémicas, amenazadas y carismáticas, con presencia de un paisaje de manglar muerto de alta productividad biológica.

El sitio cuenta con una de las mayores poblaciones de cocodrilo (Crocodylus acutus) de la costa norte de Cuba y la presencia del manatí (Trichechus manatus), especie en peligro de extinción. Se observa una población importante de delfines (Tursiops truncatus), especie carismática a escala internacional; $y$, por otra parte, el área es un corredor de aves migratorias.

\section{La comercialización sustentable}

Para lograr un desarrollo sustentable con mayor alcance es importante que el comercio actúe en beneficio de los objetivos ambientales y sociales, además de los económicos. Desde tiempo atrás, el Programa de las Naciones Unidas para el Medio Ambiente (PNUMA) trabaja con psicólogos y expertos en teorías conductistas para inducir el uso de patrones de consumo "verde" en el mundo. No obstante, se reconoce que no será suficiente para los países subdesarrollados, donde los malos hábitos de consumo se funden con la imposibilidad que enfrentan millones de personas para cubrir sus necesidades primarias. Por tanto, carece de sentido hablar de un consumo sustentable si las necesidades de consumo básicas y primarias aún no se han cubierto. 
Además, algunos países pobres, lejos de establecer alternativas culturales, económicas y ambientales que logren ser sustentables, están copiando modos de consumo de los países más ricos. Si todos los habitantes de la Tierra adoptaran el estilo de vida de los más ricos, se necesitarían los recursos de alrededor de cinco planetas para satisfacer a todos. Revertir esta situación no será fácil a pesar de los compromisos y acciones para promover el consumo sustentable.

En la Conferencia Mundial de Desarrollo Sustentable de 1992, época en que el debate sobre el financiamiento de la sustentabilidad seguía girando en torno de la ayuda financiera directa, los países en vías de desarrollo, más allá de pedir apoyo mediante la creación de un nuevo "fondo verde", solicitaron la eliminación de las barreras comerciales y un mejor acceso a los mercados -en particular para los productos agrícolas-, además de una transferencia tecnológica considerable y con condiciones preferenciales (U. S. Office of Technology Assessment, 1992).

Por su parte, los consumidores han logrado obtener cada vez más conocimientos sobre los impactos sociales y ambientales que provocan sus decisiones en el mercado, mientras que los productores se percatan con frecuencia de las posibilidades de ahorrar insumos y aumentar las utilidades gracias a la implementación de tecnología limpia; las empresas han diseñado normas ambientales y sociales, incluyendo a sus abastecedores, $y$ numerosos empresarios han asumido el desafío del rendimiento ambiental y social como un elemento de la competitividad.

Con estos elementos como telón de fondo ha tomado forma el comercio sustentable, que no es más que el nexo entre el consumo sustentable y la producción sustentable.

El comercio sustentable se define como un comercio que genera valor económico, reduce la pobreza y la desigualdad, y regenera recursos ambientales.

El comercio sustentable existe en muchos niveles y exhibe numerosos rostros: mientras que algunos ponen el énfasis en los objetivos ambientales, otros apuntan a objetivos sociales; los hay con estándares muy elevados y criterios estrictos; o bien, aquellos que han elaborado normas y criterios que pueden considerarse como primeros, segundos y terceros pasos hacia el comercio sustentable.

La comercialización puede realizarse con los valores de los humedales, donde la naturaleza y la sociedad obtienen ganancias; pero para ello es necesario estudiar, respetar y considerar la capacidad del ecosistema para no afectar su calidad ambiental o someterlo a estrés. 
Valores de la comercialización sustentable del humedal

En el siguiente apartado se analiza la comercialización sustentable en el refugio de fauna Río Máximo de Camagüey, utilizando los precios de mercado (estatal y privado). Por otra parte, se presentan otros recursos de explotación potencial para ampliar la sustentabilidad financiera del área protegida y se plantean el problema y la solución estratégica.

\section{Comercialización del flamenco}

Éste es el principal bien ambiental faunístico en el humedal. Se comercializa principalmente en el mercado internacional a través de la agencia de ventas Alcona. Este renglón exportable cuenta con una correcta diferenciación y posicionamiento en todas las áreas de destino (parques nacionales y museos, entre otros).

Los compradores extranjeros hacen su pedido a la agencia y ésta lo comunica al área protegida que es la que atiende la solicitud del cliente para hacer el envío en estricto apego a las normas técnicas. Por esta razón, existen diferencias en las cantidades vendidas en los años considerados en el análisis, así como en los ingresos financieros que se obtienen por este concepto en el humedal.

La venta promedio de flamencos para el período 1999-2003 asciende a más de 330 individuos, lo que significa un ingreso promedio superior a los $\$ 219120 \mathrm{MN}$ al año.

\section{Uso pesquero}

El uso pesquero del refugio Río Máximo, se practica tanto en la zona marina como en su zona de amortiguamiento. La entidad encargada es la Unidad Empresarial Básica (UEB) Nuevitas. En la zona de captura se pesca escama, jaiba y langosta, las cuales forman parte de la cartera de productos de la UEB para el mercado interno y externo.

Para esta actividad, la UEB cuenta con 105 embarcaciones pesqueras para la captura de langosta, ubicadas en las zonas de Punta Pepito (20 barcos pesqueros), RanchoAlegre ( 15 gomas), Cabilla ( 20 gomas), Bocaina (30 gomas) y La Mojarra (20 gomas).

Al no existir estudios referidos a la capacidad máxima de explotación del área, ésta respeta los resultados de los muestreos biológicos. Los parámetros 
a considerar son las tallas mínimas legales así como el comportamiento (fluctuación) que mantienen las tallas de las diferentes especies en el período determinado y de la captura, en sentido general.

Estas zonas poseen restricciones por las acciones aprobadas en los planes de manejo y en los planes operativos que están conciliados con el Ministerio de Pesca, con el objetivo de armonizar ambos intereses; entre éstos se encuentran la comercialización sustentable y el uso fundamental en la zona de pesca, que consiste en alimentar a los diferentes individuos de la fauna residente $y$ otras que consumen y descansan para continuar viajes hacia otros destinos.

\section{Pesca estatal por especies}

En la información estadística y en las entrevistas se obtuvieron datos acerca del nivel de captura para este sector económico por especies, así como del precio de su comercialización en el período 1999-2003.

\section{a) Langosta}

Esta especie de alta demanda no se captura en los meses de febrero, marzo, abril y mayo, por encontrarse en veda reproductiva. Es notable además que no existan diferencias significativas en los volúmenes de captura entre un mes y otro por las razones antes señaladas. El nivel de captura para el período analizado tiene un promedio de $9500 \mathrm{~kg}$ anuales. El precio de la comercialización de esta especie oscila entre los 15 y 24 dólares por kilogramo.

b) Jaiba

A diferencia de las otras especies, la jaiba se pesca durante todo el año, respetando las restricciones para la sustentabilidad. El comportamiento de la captura no posee grandes diferencias entre sus períodos, ya que se encuentra entre los 12000 y 30000 kilogramos al año. El precio de la comercialización de esta especie para el productor es de 1.60 dólares el kilo.

\section{c) Escama}

En los volúmenes de captura de la especie también se aprecia un comportamiento mensual similar. Sólo en febrero, marzo y abril se permite la pesca de escama, fundamentalmente macabí y mojarra, por lo que durante el resto del año estas especies se encuentran en veda. 
El precio de comercialización de la especie para la entidad económica estatal, oscila entre los 10 y los 13 pesos MN el kilo y el nivel de explotación comprende entre los 1800 y $2000 \mathrm{~kg}$ al año.

En resumen, para calcular el valor económico directo por concepto de pesca sustentable de las especies antes referidas, procede demostrar que no existen diferencias significativas en los volúmenes capturados en el período de análisis, utilizando el análisis de varianza de un solo factor:

CuAdro I. Resumen del análisis de VARIANZA POR ESPECIE

\begin{tabular}{lcc}
\hline Especie & Valor de F & Probabilidad \\
\hline Langosta & 151.5199793 & $1.3943 \mathrm{E}-2 \mathrm{I}$ \\
Jaiba & 1191.872197 & $1.04726 \mathrm{E}-52$ \\
Escamas & 30 & $1.51239 \mathrm{E}-05$ \\
\hline
\end{tabular}

Fuente: Elaboración propia.

De acuerdo con la probabilidad obtenida en todos los casos, no se manifiestan diferencias significativas en la captura por especies entre un año y otro; excepto en el período 200I, en el que se registra un descenso en los niveles debido a la falta de recursos para el mantenimiento de embarcaciones (reparación de roturas) y del parque de artes de pesca, que se encuentra en mal estado.

\section{Cálculo de la captura promedio anual por especies}

El valor promedio permite conocer cuál ha sido el comportamiento de la captura en las zonas del refugio y amortiguamiento durante los cinco años que se toman como referencia.

Al considerar los resultados obtenidos, el promedio resulta representativo para calcular posteriormente el valor de cada especie, por cuanto no existen variaciones significativas en los promedios de captura de cada una de las especies, condicionado fundamentalmente por la pequeña variación en los volúmenes de captura en cada uno de los meses. 


\section{Estimación de los ingresos anuales por especies}

La determinación del valor promedio anual por especie se realiza a través de la multiplicación de los volúmenes de captura de cada una de las especies, por el precio específico de comercialización (mercado interno y externo) empleando el precio de venta del tenente.

Para la langosta, el valor promedio anual se encuentra entre los $\$ 142800$ y $\$ 228480$ cuc; por concepto de jaiba, un promedio anual superior a los $\$ 28000$ CUC;y en escamas, entre $\$ 17000$ y $\$ 22100$ MN. Este segmento de la comercialización tiene como destino fundamental el consumo poblacional y social.

\section{Pesca privada}

Limítrofe al área protegida se ubican dos asentamientos poblacionales: Alfredo Álvarez Mola y Gurugú, con 5 I 2 y 375 habitantes, respectivamente. En el trabajo de campo realizado en estas comunidades se pudo obtener la información necesaria para calcular el nivel de captura privada en la zona objeto de estudio. La administración del refugio otorga permiso para actividades de uso consuntivo (consumo familiar), siempre que se respeten las restricciones planteadas en el plan de manejo y en el plan operativo. Sin embargo, se aprecia la existencia de pesca ilegal por parte de los residentes, principalmente de Gurugú y de la población flotante de la microrregión.

Una parte de la captura se destina a la comercialización privada en la comunidad para satisfacer las necesidades de otros productos.

Según el clima, la población residente captura cuatro quintales de pescado por jornada (principalmente mojarra, liseta y lisa) durante diez días como promedio por mes (entrevistas a los pescadores de las dos comunidades).

Para calcular el valor se aplica el procedimiento siguiente:

- El cálculo de la conversión del quintal en libras es de 4000 libras mensuales, lo que equivale a 48000 libras al año.

- A este valor se le aplica 70\% para calcular el volumen de masa en libras (según criterio del experto), lo que representa alrededor de 33600 libras anuales.

- En la comunidad, el precio de venta de los privados, para estas especies, tiene un promedio de $\$ 5.00$ la libra (entrevistas a los habitantes de las dos comunidades). 
- La parte destinada al propio consumo familiar (6000 libras anuales) significa un gasto evitado, por lo que se le considera un ingreso.

Entonces, de acuerdo con lo anterior es posible plantear que:

- Con la venta del pescado al precio establecido por el mercado local, las personas que se dedican a esta actividad obtienen un ingreso estimado de $\$ 138000$, y a su vez un ahorro al no tener que comprarlo para su consumo en un precio de $\$ 30000$.

- A partir del análisis realizado,y tomando como referencia el promedio anual, se calcula un valor total para la actividad pesquera privada en la zona marina del refugio, así como en su zona de amortiguamiento.

Finalmente, se calcula el beneficio económico total por concepto de pesca en el humedal Río Máximo de Camagüey. Para la langosta y las escamas se emplea el promedio entre los dos tipos de precios (mínimos y máximos).

Cuadro 2. Estimado de ingreso para el uso pesquero en un año ( $\$$ CUC y mN)

\begin{tabular}{lcc}
\hline Especie & \multicolumn{2}{c}{ Tipo de moneda } \\
& CUC & MN \\
\hline $\begin{array}{l}\text { Langosta estatal } \\
\text { Escamas estatal } \\
\text { Jaiba estatal }\end{array}$ & 185640 & 19500 \\
Pesca privada & 28480 & 168000 \\
\hline Total & $\$ 214120$ & $\$ 187500$ \\
\hline
\end{tabular}

Fuente: Elaboración propia.

Si se utiliza la conversión del dólar en moneda nacional, el valor total de la pesca en la zona de estudio es de \$40I 620 en un año.

El área protegida tiene otros ingresos por la comercialización de madera y posturas de plantas. En el primer caso, por la venta de leña o bolos de madera preciosa a la empresa forestal, entre otros usuarios, producto del raleo para mantener la salud del bosque; en el segundo, por posturas según pedido del cliente con fines de reforestación en el territorio y fuera de éste.

Estos ingresos constituyen también una vía de sustentabilidad financiera y su importe asciende a $\$ 3$ I 973.85 en posturas y $\$ 270773.34$ en madera para el año de referencia (Balance de estado de resultado del refugio, 2003). Estas partidas no se contabilizan en períodos anteriores. 
A continuación, se agregan los diferentes conceptos para expresar el total de la comercialización sustentable en el ecosistema en cuestión. El cuadro 3 muestra el resumen.

CUADRO 3. EstimAdo DE INGRESO TOTAL EN UN AÑO

\begin{tabular}{lr}
\hline Elementos & Ingresos en MN (\$) \\
\hline Pesca en ambos sectores & 40 I 620.00 \\
Flamenco & 219 I20.00 \\
Posturas de la flora & 31973.85 \\
Madera & 270773.34 \\
\hline Total & 923487.19 \\
\hline
\end{tabular}

Fuente: Elaboración propia.

\section{Análisis de las debilidades, amenazas, fortalezas y oportunidades del del refugio de fauna}

A partir de la diferenciación del producto y su posicionamiento en el mercado, de la percepción de los consumidores y la competencia, se obtiene que el humedal dispone de otros potenciales que pueden enriquecer la sustentabilidad financiera. La diferenciación consiste en determinar las características que distinguen la oferta comercial y cómo contribuyen a que sea percibida como distinta de las demás en el mercado interno y externo.

Se desarrolla una mesa de trabajo con una convocatoria a los autores del plan de manejo y del plan operativo del sitio para desarrollar una tormenta de ideas que identifique a los factores, tanto internos como externos, que serán utilizados posteriormente en la matriz DAFO. La selección de los integrantes de la mesa de trabajo responde a la necesidad de imprimir mayor realidad al ejercicio.

Una vez identificados esos factores, se procede a la ponderación individual con los integrantes para excluir los factores de menor importancia, los que se resumen de la siguiente forma:

Fortalezas

I. El mayor sitio de nidificación del flamenco rosado en la región del Caribe

2. Una reserva de biodiversidad

3. Un sitio Ramsar

4. Un polígono de manejo sustentable 


\section{Debilidades}

I. Insuficiente desarrollo del programa de turismo científico y de las actividades de investigación

2. Lejanía, difícil acceso y condiciones adversas del área protegida

3. Carencia de infraestructura para logística

4. Insuficiente personal científico y técnico

Oportunidades

I. Convocatorias de financiamiento por parte de proyectos internacionales para el manejo de áreas protegidas en el país y en el mundo

2. Intercambios y convenios con organismos internacionales

3. Demanda de turismo científico y especializado en el mundo

4.Apoyo del Estado cubano para el desarrollo turístico

Amenazas

I. Cambios climáticos

2. Actividades económicas no sustentables en la cuenca hidrográfica

3. Otras áreas en Cuba con importantes valores naturales

4. Cambios en el uso del suelo en el área de influencia del área protegida

Definidas las oportunidades, amenazas, fortalezas y debilidades se procede de forma individual a su evaluación, utilizando una escala de valores donde 3 es fuerte, 2 es moderado y I es débil.

Al confeccionar la matriz se observó una clara concentración de los impactos en el cuadrante donde se cruzan las fortalezas y oportunidades, por lo que se propone seguir una estrategia ofensiva que indica las acciones encaminadas para lograr el liderazgo del mercado, sus acciones de crecimiento, así como acciones de desarrollo de nuevos productos y otros mercados.

Analizados los resultados obtenidos, se identifican los factores de éxito y fracaso, las oportunidades de mayor aprovechamiento, así como las amenazas que pueden ser mejor contrarrestadas.

Factores de éxito

I. Mayor sitio de nidificación del flamenco rosado en la región del Caribe 2. Polígono demostrativo de manejo sustentable 
Factores de fracaso

I. Insuficiente desarrollo del programa de turismo científico y de las actividades de investigación

2. Insuficiencia del personal científico y técnico

Oportunidades con posibilidad de ser mejor aprovechadas

I. Existencia de proyectos internacionales para el manejo de áreas protegidas en el país y en el mundo

2. Convenios con organismos internacionales

Amenazas que pueden ser mejor contrarrestadas

I. Cambios en el uso del suelo en el área de influencia del área protegida

2. Cambios climáticos

\section{Problema estratégico}

En el potencial para la comercialización sustentable están influyendo los cambios en el uso del suelo y climáticos. Si no se resuelve el insuficiente desarrollo del programa de turismo científico y actividades de investigación, así como la insuficiencia de personal calificado, aunque se cuente con el mayor sitio de nidificación del flamenco rosado en la región caribeña y sea un polígono demostrativo de manejo sustentable, no se podrán aprovechar los proyectos internacionales para el manejo de áreas protegidas y los convenios con organismos internacionales.

\section{Solución estratégica}

En el potencial para la comercialización sustentable está influyendo la existencia de proyectos internacionales para el manejo de áreas protegidas y los convenios con organismos internacionales. Si se toma en cuenta que el refugio es el sitio de mayor nidificación del flamenco rosado en la región del Caribe y que es polígono demostrativo de manejo sustentable y, por otra parte, si se reducen los factores de fracaso, esto es, el insuficiente desarrollo del programa de turismo científico, las actividades de investigación, así como la insuficiencia de personal calificado, se estará en mejores condiciones para enfrentar los cambios del uso del suelo y climáticos. 


\section{Conclusiones}

Utilizando la base de datos del período 1999-2003, el cálculo de la comercialización sustentable para el refugio de fauna Río Máximo de Camagüey se aproxima al millón de pesos en un año. Estos ingresos pueden ser superados al demostrarse que otro potencial puede ser explotado utilizando como base el principio de la sustentabilidad.

\section{FUENTES CONSULTADAS}

Blas, L. (1998). Parques nacionales iberoamericanos. Madrid: Ediciones Anaya. Bosch, R. et al. (1998). Turismo y medio ambiente. Madrid: Centro de Estudios Ramón Aceres, 89 p. (Colección CEURA).

Bruce, M. ( 1999). La gestión de los recursos y del medio ambiente. Madrid: Ediciones Mundi Prensa, 290 p.

Cals, J. (2002). Evaluación de proyecto. Análisis de la rentabilidad social desde la perspectiva del turismo y el ocio. España: Universidad Autónoma de Barcelona.

Cherni, J. (200I).“Medio ambiente y globalización: desarrollo sustentable modernizado”. Revista Economía y Desarrollo, 31, I29, 193-212.

Delgado, C. (1999). Cuba verde. En busca de un modelo para la sustentabilidad en el siglo XXI. La Habana: Editorial José Martí / Instituto del Libro.

Gudynas, E. (2000). Los límites de la sustentabilidad débil y el tránsito desde el capital natural al patrimonio ecológico. Dirección General Sectorial de Educación Ambiental y Participación Comunitaria del Ministerio del Ambiente y de los Recursos Naturales, 4, II, 7-I I.

Lambert,A. (2003). Valoración económica de los humedales: un componente importante de las estrategias de gestión de los humedales a nivel de las cuencas fluviales. Documento inédito, mayo de 2003.

Llanes, J. (1999). "La valoración económica de los recursos ambientales: entre lo novedad y la duda". Revista Economía y Desarrollo, 2, I83-198.

Romano,J.(2000). Desarrollo sostenible y evaluación ambiental. Del impacto al pacto con nuestro entorno. Madrid: Ámbitos Ediciones, 320 p.

U.S. Office of Technology Assessment (1992). Trade and the environment:conflicts and opportunities. Washington, D.C.: Government Printing Office.

Wautiez, F.y Reyes, B. (200 I). Indicadores locales para la sustentabilidad. La Habana: Instituto de Ecología / Publicaciones Acuario, I 36 p. 